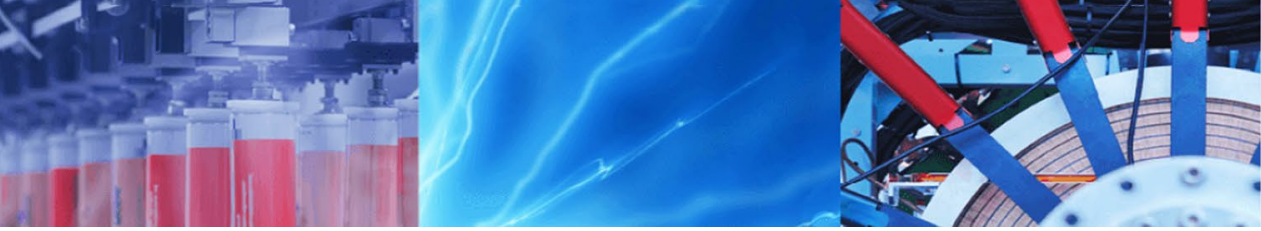

Research Article

\title{
A proposed method of sample preparation and homogenization of hemp for the molecular analysis of cannabinoids
}

\author{
Zachary P. Morehouse ${ }^{1,2,3}$ (D) Gabriella L. Ryan ${ }^{1,4} \cdot$ Caleb M. Proctor $^{1,4} \cdot$ Akelachi Okparanta $^{1} \cdot$ Will Todd $^{1}$. \\ Derrick Bunting ${ }^{1} \cdot$ Taylor White $^{1,4} \cdot$ Steven Parez ${ }^{5} \cdot$ Blessida Miller $^{5} \cdot$ Veronica Colon ${ }^{5} \cdot$ Brandon Easparro $^{1}$. \\ James Atwood ${ }^{1}$. YoungChul Park ${ }^{5}$ Rodney J. Nash ${ }^{1,3,4}$
}

Received: 22 March 2021 / Accepted: 27 July 2021

Published online: 31 July 2021

(c) The Author(s) $2021 \quad$ OPEN

\begin{abstract}
The use of Cannabis sativa, or Hemp, in commercial, recreational, and pharmacological applications is on the rise in the United States and worldwide. Many of these applications have guidelines associated with them dependent on the concentration of cannabinoid molecules that keep the products classified as hemp versus marijuana or that allow the producer to comment on the purity and potency of their product. Herein, we propose a method for homogenization of hemp that results in small particle sizes, uniform samples, and does not alter the cannabinoid concentrations during processing, allowing for optimal and reproducible potency testing. Using a novel "active grinding media" we homogenized commercially available hemp to analyze approximately $100 \mathrm{mg}$ samples of homogenate via sieve analysis and highperformance liquid chromatography to assess the resulting size and potency of the sample when using this methodology. When processing hemp samples with our proposed methodology, we have demonstrated the ability to produce $60.2 \%$ of all particles $<1.25 \mathrm{~mm}$ with increased cannabinoid recovery compared to homogenates with larger average particle sizes. Maintaining sample temperatures below $35^{\circ} \mathrm{C}$ during processing, we showed that our method does not thermally induce decarboxylation reactions that would result in major cannabinoid profile changes. We have developed a method for hemp processing via homogenization that does not alter the cannabinoid profile during processing, while consistently producing small particle sizes in a uniformly processed sample. This method allows for optimal and reproducible hemp processing when evaluating hemp and hemp-based products being brought to commercial markets.
\end{abstract}

Keywords Hemp · Hemp processing · Hemp grinding · Homogenization - Sample preparation · Cannabinoid detection . $\mathrm{THC} \cdot \mathrm{CBD}$

\section{Abbreviations}

THCA $\quad \Delta^{9}$-Tetrahydrocannabinolic acid

THC Tetrahydrocannabinol

CBDA Cannabidiolic acid

CBD Cannabidiol

HPLC High performance liquid chromatography

\section{Background}

Cannabis sativa, commonly known as "hemp", has widestretching usages in industrial, medicinal, and agricultural applications across the world [1]. Currently the applications of both hemp and marijuana are being explored in greater

Supplementary Information The online version contains supplementary material available at https://doi.org/10.1007/s42452-02104747-6.

$\triangle$ Rodney J. Nash, rnash@omni-inc.com | ${ }^{1}$ Omni International, Inc, 935 Cobb Place Blvd, Kennesaw, GA, USA. ${ }^{2}$ Michigan State University College of Osteopathic Medicine, East Lansing, MI, USA. ${ }^{3}$ Jeevan Biosciences, Tucker, GA, USA. ${ }^{4}$ Department of Biology, Georgia State University, Atlanta, GA, USA. ${ }^{5}$ AMERICANNA Laboratories, LLC, Jacksonville, FL 11757, USA.

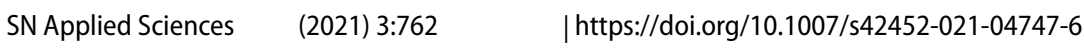


detail than previously seen by pharmaceutical and therapeutic applications [2-4]. The naturally occurring cannabinoid molecules found in hemp are the basis for its pharmacologic activity and viewed as a presumptive area of increasing importance in the field of pharmacology [2, 3]. In addition to the medical application of cannabinoid-based compounds, the use of cannabinoid infused products is on the rise for recreational usage [5].

As states across the United States begin to legalize the sale and usage of marijuana and cannabinoid derived products, there is an increased longing for products that will induce the reported psychoactive or therapeutic effects of these cannabinoids $[2,5,6]$. With demand for these products exponentially rising, the hemp industry has grown to a billion-dollar industry crossing recreational and medicinal markets for cannabinoids and cannabinoid-derived products [5]. However, as the industry has dramatically expanded in recent years, regulations and best-practice methods have not been keeping up [6]. Currently, there are a variety of unregulated techniques used in hemp processing for sample preparation and the assessment of products for cannabinoid concentrations.

Testing for the cannabinoids, $\Delta^{9}$-tetrahydrocannabinolic acid (THCA) and cannabidiolic acid (CBDA), and their decarboxylated counterparts, $\Delta^{9}$-tetrahydrocannabinol ( $\Delta^{9}$-THC) and cannabidiol (CBD), are critical in evaluating hemp-based products being brought to market [7]. In the US, hempbased products must remain under certain concentrations of THCA and THC to remain labeled as hemp versus marijuana, a key determination in many areas given the legality of hemp versus marijuana in many municipalities [8]. Additionally, the CBDA and CBD concentrations within products have been related to their analgesic potency, thus accurate measurement and reporting of these values are vital to proper labeling and pricing of these products to protect both the supplier and consumer [6-8].

Herein, we report a method for sample preparation of hemp involving bead-mill based homogenization using a novel "active grinding media" that provides uniform processing of hemp samples with reproducible particle sizes and no alterations to the carboxylation states of THCA or CBDA. This method allows for accurate potency testing of hemp and hemp-based products through a homogenization that results in a completely processed, homogenous sample.

\section{Methods}

\subsection{Sample preparation and homogenization}

To measure cannabinoid potency, commercially available hemp was purchased. Hemp flower was placed in a
$50 \mathrm{~mL}$ conical tube (Omni International, Cat. No. 19-6650), followed by the placement of the active grinding media (Omni International, Cat. No. 19-900 M) specifically for hemp. Tubes were loaded into the BR96 homogenizer (Omni International, Cat. No. 27-0001) in an even parallel series $(2,4,6$, or 8 tubes). Once loaded, the BR96 homogenizer was operated at $25 \mathrm{~Hz}$ for 1 cycle (15 s), 2 separate cycles (total of $30 \mathrm{~s}$ ), 4 separate cycles (total of $60 \mathrm{~s}$ ), and 6 separate cycles (total of $90 \mathrm{~s}$ ).

\subsection{Sieve analysis}

A diamond tap sieves shaker (Esslinger.com, Cat. No. 51.0569) was used. Tap sieve shakers are specified in various standards for particle size analysis. The shaker was used in a horizontal and circular motion, and superimposed by a vertical motion while administering a taping action. Tap sieve shakers was specified for specific particle size analysis ( $1.10 \mathrm{~mm}$ to $4.50 \mathrm{~mm})$. Starting weight before and after analysis was recorded and distribution percentages were calculated.

\subsection{Cannabinoid sample analysis for potency}

Approximately $100 \mathrm{mg}$ of homogenized flower matrix was isolated and $5 \mathrm{~mL}$ of methanol was added. Sample was sonicated, vortexed and centrifugated. Supernatant was diluted with $\mathrm{MeOH} / \mathrm{H}_{2} \mathrm{O}(80 / 20, \mathrm{v} / \mathrm{v})+0.1 \%$ formic acid as appropriate for injection. Sample was injected (5 $\mu$ l) into the Agilent 1220 Infinity II LC. The analytical column used was the Infinity Poroshell $120 \mathrm{EC}-\mathrm{C} 18,3.0 \mathrm{~mm} \times 50 \mathrm{~mm}$, $2.7 \mu \mathrm{M}$ at $50{ }^{\circ} \mathrm{C}$. Gradient used was 0 to $1 \mathrm{~min}, 60 \% \mathrm{~B}$, $7-8.2 \mathrm{~min}, 60$ to $77 \% \mathrm{~B}$, and 8.2 to $10 \mathrm{~min}, 95 \% \mathrm{~B}$.

\subsection{Cannabinoid standards}

Standards for THC, THCA, CBD and CBDA ( $1 \mathrm{mg} / \mathrm{ml})$ were obtained from Cerilliant (Round Rock, Texas). Standards were combined and a stock solution with a concentration of $250 \mathrm{ppm}$ for each cannabinoid was made and serially diluted in $100 \%$ methanol following the dilution series in Table 1. Fifteen $\mu \mathrm{L}$ of each dilution was diluted further with $35 \mu \mathrm{Ls}$ of $100 \%$ methanol and analyzed by reverse phase HPLC.

\subsection{Sample preparation}

The six ( $1 \mathrm{~mL}$ samples of the 250-ppm cannabinoid) standards were added to reinforced tubes $(2 \mathrm{~mL})$ that were prefilled with $2.8 \mathrm{~mm}$ ceramic beads (Omni International, Kennesaw, GA, Cat. No. 19-628). The tubes were processed on the Bead Ruptor Elite Bead Mill Homogenizer at $5 \mathrm{~m} / \mathrm{s}$ for increasing durations of $10,20,30,60$, and $120 \mathrm{~s}$. After each 
Table 1 Particle size distribution following hemp homogenization as tested for via particle sieves

\begin{tabular}{|c|c|c|c|c|c|}
\hline Parameter & Particle size distribution & $\begin{array}{l}\text { Weight sieve } 2 \\
\mathbf{1 . 2 5} \mathbf{~ m m}\end{array}$ & $\begin{array}{l}\text { Weight sieve } 7 \\
\mathbf{1 . 8 8} \mathbf{~ m m ~}\end{array}$ & $\begin{array}{l}\text { Weight sieve } 12 \\
\mathbf{2 . 8 7} \mathbf{~} \mathbf{~ m}\end{array}$ & $\begin{array}{l}\text { Weight sieve } 17 \\
\mathbf{3 . 8 6} \mathbf{~ m m}\end{array}$ \\
\hline \multirow[t]{2}{*}{ Set-1 } & $1.009 \mathrm{~g}(\mathrm{n}=3)$ & 0.245 & 0.357 & 0.3 & 0.112 \\
\hline & Distribution (\%) & 24.4 & 35.5 & 29.0 & 11.2 \\
\hline \multirow[t]{2}{*}{ Set-2 } & $1.006 \mathrm{~g}(\mathrm{n}=3)$ & 0.490 & 0.414 & 0.069 & 0.035 \\
\hline & Distribution (\%) & 48.7 & 41.2 & 6.9 & 3.5 \\
\hline \multirow[t]{2}{*}{ Set-3 } & $1.007 \mathrm{~g}(\mathrm{n}=3)$ & 0.642 & 0.238 & 0.081 & 0.043 \\
\hline & Distribution (\%) & 63.85 & 23.69 & 8.08 & 4.24 \\
\hline \multirow[t]{2}{*}{ Set-4 } & $1.007 \mathrm{~g}(\mathrm{n}=3)$ & 0.606 & 0.268 & 0.106 & 0.025 \\
\hline & Distribution (\%) & 60.239 & 26.673 & 10.537 & 2.485 \\
\hline
\end{tabular}

For each listed set, three samples $(n=3)$ were utilized at the listed weight and the readings at each weight sieve were then averaged across all three samples to provide the values listed in this table. $(n=3$ STD $\leq \pm 0.05)$

time point, $250 \mu \mathrm{L}$ was removed to a new $1.5 \mathrm{~mL}$ microcentrifuge tube and placed on ice. As a positive control, $500 \mu \mathrm{L}$ of the 250-ppm cannabinoid standard was placed in a $1.5 \mathrm{~mL}$ microcentrifuge tube and heated, at $90^{\circ} \mathrm{C}$, for $180 \mathrm{~min}$. All samples were filtered through a $0.2 \mu \mathrm{m}$ spin filter and $15 \mu \mathrm{L}$ of the filtrate was combined with $35 \mu \mathrm{L}$ of $100 \%$ HPLC grade methanol for reverse phase HPLC analysis.

\subsection{Reverse phase HPLC for standards}

Cannabinoid separation and quantification was performed on a Waters 1525 HPLC (Waters Corp, Milford, MA) equipped with a binary pump and 2996 photodiode array detector. Buffer $\mathrm{A}$ consisted of $\mathrm{dd}_{2} \mathrm{O}$ and formic acid $(0.2 \% \mathrm{v} / \mathrm{v})$ and buffer $\mathrm{B}$ was Acetonitrile and formic acid $(0.2 \% \mathrm{v} / \mathrm{v})$. Fifteen $\mu \mathrm{L}$ of each sample was separated on a Raptor ARC-18 $150 \mathrm{~mm} \times 4.6 \mathrm{~mm}, 2.7 \mu \mathrm{m}$ column over a $20-$ min linear gradient from $60 \%$ B to $100 \%$ B at a flow rate of $1.5 \mathrm{~mL} / \mathrm{min}$. Absorbance was measured at $280 \mathrm{~nm}$.

\section{Results}

Through the homogenization methods described above, we have shown that our methodology results in homogenate particle sizes $<1.25 \mathrm{~mm}$. While running one cycle of our optimized homogenization parameters yielded $11.2 \%$ of particles $<1.25 \mathrm{~mm}$; we observed that with each additional cycle of 15 s of homogenization, we were able to increase the percentage of homogenate particles $<1.25 \mathrm{~mm}$ to as high as $60.2 \%$ of all particles within the sample after four cycles of $15 \mathrm{~s}$ (Table 1). This data suggests that intermittent homogenization produces a complete homogenate with the average particle size decreasing after each cycle. As average particle size decreased with each cycle of homogenization, we also observed an increased extraction of
CBD, CDBA, THC, and THCA, with a significant increase in the detection of CBD and CBDA (Table 2,). These findings are depicted in the resulting average concentration of each cannabinoid and as the relative extraction efficiency, examining the difference in recovered cannabinoids after each homogenization parameter when compared to set-1.

In addition to providing particle sizes $<1.25 \mathrm{~mm}$, the use of our active grinding media and cyclical homogenization approach did not cause decarboxylation changes to the THCA and CBDA populations seen in the hemp tested as confirmed by reverse phase HPLC (Fig. 1, Supplemental Fig. 1). Processing hemp samples under these parameters did not produce enough thermal energy to alter the carboxylation start of the major cannabinoids tested for. Maintaining temperatures during sample processing under $90{ }^{\circ} \mathrm{C}$ is the benchmark to ensure that cannabinoids being tested for downstream do not have their concentrations altered during processing via decarboxylation reactions (Fig. 2). Additional homogenization runs with extended run times, up to $180 \mathrm{~s}$, were also run in an attempt to determine the processing time required to generate enough heat to induce the decarboxylation reactions that convert THCA to THC and CBDA to CBD (Fig. 1, Supplemental Fig. 1). These extended time homogenizations showed that our method maintains processing temperatures under $35^{\circ} \mathrm{C}$, when measuring the temperature both internal and external to tubes used for the sample processing throughout the run (Fig. 3).

\section{Discussion}

The method proposed in this manuscript for hemp sample preparation via homogenization with our novel active grinding media, demonstrates a critical step forward in the commercial and medical hemp markets. This methodology brings forward a standardized protocol for hemp 
Table 2 Overall extraction efficiency of major cannabinoids based off homogenization parameters

\begin{tabular}{|c|c|c|c|c|c|c|c|c|}
\hline \multirow{2}{*}{$\begin{array}{l}\text { Homogeniza- } \\
\text { tion param- } \\
\text { eters }\end{array}$} & \multicolumn{2}{|c|}{ Cannabidiolic acid } & \multicolumn{2}{|l|}{ Cannabidiol } & \multicolumn{2}{|c|}{$\Delta 9$-Tetrahydrocannabinol } & \multicolumn{2}{|c|}{ Tetrahydrocannabinolic acid } \\
\hline & $\begin{array}{l}\text { Average } \\
\text { concentra- } \\
\text { tion (\%) }\end{array}$ & $\begin{array}{l}\text { Relative } \\
\text { extraction } \\
\text { efficiency (\%) }\end{array}$ & $\begin{array}{l}\text { Average } \\
\text { concentra- } \\
\text { tion (\%) }\end{array}$ & $\begin{array}{l}\text { Relative } \\
\text { extraction } \\
\text { efficiency (\%) }\end{array}$ & $\begin{array}{l}\text { Average } \\
\text { concentra- } \\
\text { tion (\%) }\end{array}$ & $\begin{array}{l}\text { Relative } \\
\text { extraction } \\
\text { efficiency (\%) }\end{array}$ & $\begin{array}{l}\text { Average } \\
\text { concentra- } \\
\text { tion (\%) }\end{array}$ & $\begin{array}{l}\text { Relative } \\
\text { extraction } \\
\text { efficiency (\%) }\end{array}$ \\
\hline Set-1 & 5.47 & 0.0 & 1.92 & 0.0 & 0.16 & 0.0 & 0.15 & 0.0 \\
\hline Set-2 & 5.54 & 1.3 & 1.96 & 2.1 & 0.16 & 0.0 & 0.15 & 0.0 \\
\hline Set-3 & 5.58 & 2.0 & 2.22 & 15.6 & 0.16 & 0.0 & 0.17 & 13.3 \\
\hline Set-4 & 6.34 & 15.9 & 2.36 & 22.9 & 0.20 & 25.0 & 0.16 & 6.7 \\
\hline
\end{tabular}

Set-1, $5 \mathrm{~m} / \mathrm{s}$ for $30 \mathrm{~s}$. Set-2, $5 \mathrm{~m} / \mathrm{s}$ for $60 \mathrm{~s}$. Set-3, $5 \mathrm{~m} / \mathrm{s}$ for $90 \mathrm{~s}$. Set- $4,5 \mathrm{~m} / \mathrm{s}$ for $120 \mathrm{~s}$. For each listed set, three samples $(\mathrm{n}=3)$ were utilized at the listed weight and cannabinoid concentration of each were then averaged across all three samples to provide the values listed in this table. $(\mathrm{n}=3$ STD $\leq \pm 0.05)$

Fig. 1 Reverse HPLC results visualizing CBDA, CBD, THCA, and THC concentrations as absorbance units following hemp homogenization (green), $90 \mathrm{C}$ heating of sample (black), and no sample preparation (blue)
Fig. 2 CBD, CBDA, THC and THCA structure. Acid forms (CBDA and THCA) are decarboxylated to neutral forms when exposed to temperatures in excess of $90^{\circ} \mathrm{C}$, altering the cannabinoid profile of the processed hemp

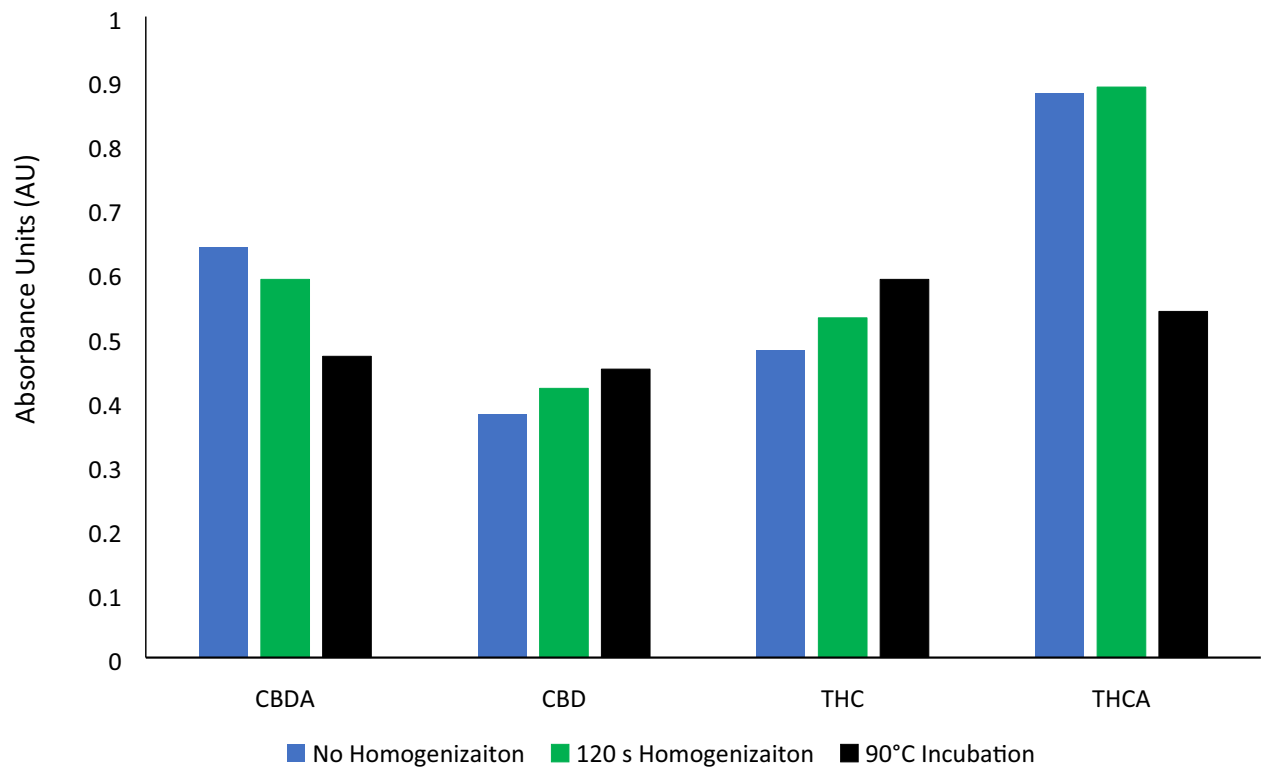<smiles>C=C(C)C1CCC(C)=C[C@H]1c1c(O)cc(CCCCC)c(C(=O)O)c1O</smiles>

Cannabidiolic Acid (CBDA)<smiles>CCCCCc1cc2c(c(O)c1C(=O)O)[C@@H]1C=C(C)CC[C@H]1C(C)O2</smiles>

Tetrahydrocannabinolic Acid (THCA)
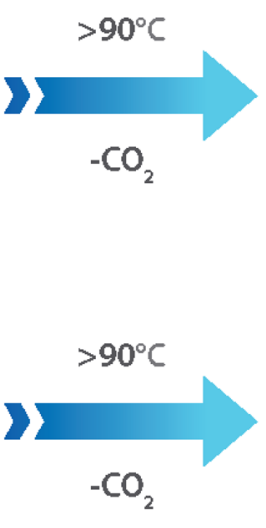<smiles>C=C(C)[C@H]1C=C(C)CC[C@H]1c1c(O)cc(CCCCC)cc1O</smiles><smiles>CCCCCc1cc(O)c2c(c1)OC(C)[C@H]1CCC(C)=C[C@H]21</smiles>

Tetrahydrocannabinol (THC) 
Fig. 3 Temperature of homogenization tubes internally and externally was measured throughout the homogenization process. Note that the homogenization tubes never surpassed $35 \mathrm{C}$, maintaining the cannabinoids well under the $90 \mathrm{C}$ benchmark that causes conversion from the acidic to neural forms

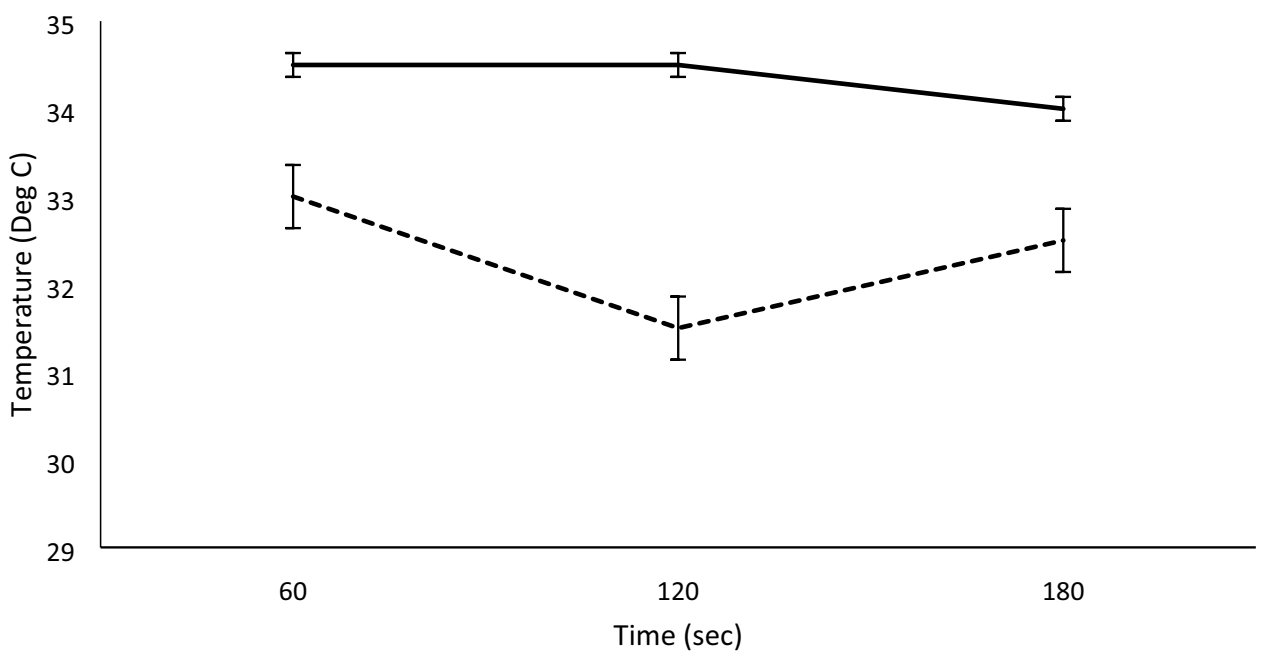

- Average Tube Internal Temperature - - - - Average Tube External Temperature processing that allows for reproducible results, small particle sizes, and unaltered cannabinoid profiles, all of which are essential as the market expands and inevitably becomes more standardized and regulated. While it is no surprise that federal, state, and local regulations have not developed as rapidly as the marked for hemp-based products has, we must be fully aware that these regulations are coming and work proactively to establish robust industry standard methods for processing and testing hemp involved products.

The authors of this study firmly believe that this processing methodology is a strong first step towards establishing those industry standards for hemp processing and testing. Current guidelines surrounding hemp derived products require that the measured THC levels remain below certain prescribed limits in order to remain labeled as hemp versus marijuana-a critical distinction in many markets across the United States where hemp is legal and marijuana is not $[6,8]$. While some argues that this is a game of legal semantics, this paradigm is critical for the producers of these products and compliance with these regulations is directly impacting their ability to remain in business. With such high stakes involved in accurate cannabinoid profiling, we were surprised to see so much variability in sample preparation techniques for these processes. The use of coffee grinders, blenders, and other various dissociation devices have two major problems seen when they are utilized for hemp sample preparation prior to cannabinoid profiling - their dissociation processes are highly energetic, potentially heating the sample to a temperature to induce decarboxylation of THCA to THC, and they are often very difficult to clean, allowing for cross contamination between samples [9-11]. Both of these are ameliorated with our proposed methodology, implying a superior process for sample preparation, robust enough to be implemented into a standardized regulatory workflow.

However, we do acknowledge that given the potential hundreds of coffee grinders and blenders in the current market which can be utilized for cannabis homogenization purposes. It would be impossible to compare each of them to our proposed methodology or even determine which is considered an "industry leader" brand in this market given the wide variability of techniques and lack of regulation and as such we have elected to avoid direct comparisons with this proposed methodology. This purposeful decision of omission does not impact the utility of the proposed technology for hemp processing, yet further supports the need of a standardization of processing techniques to allow for future studies to benchmark against.

In addition to the critical need for a standardized process that will not impact the THC profile of a hemp sample, it is important that the CBD profile be measured accurately as well $[6,8]$. The CBD profile of a hemp-based product is often tied to the potency of the product, with higher concentrations of CBD fetching higher prices in the market. Ensuring that accurate measurement of these cannabinoids is done it requires a process that does not induce excessive thermal energy to the sample and produces a reliably small particle size throughout the sample to ensure accurate measurement with HPLC.

As the hemp market continues to exponentially grow across the US, we feel that it is a pertinent time to adopt industry standardized procedures. The significance of this work is to show that homogenization is a dependable and consistent method for measuring cannabinoid potency in hemp. With many states allowing hemp (less than $0.3 \% \mathrm{THC}$ ) usage but prohibit the use of cannabis with high levels of THC, it is critical to develop processing 
technologies that will not alter these cannabinoid profiles. The conversion of THCA to active THC can redefine a hemp product, into a high THC cannabis product, rendering it illegal. Having an accurate measurement of all forms of cannabinoids is important to prevent this and we show that the smaller the particles of hemp the more accurate potency testing becomes. Furthermore, this method is repeatable and no contamination from multiple samples occurs. Having accurate potency testing also helps growers market their premium product verses product of lesser grades of potency. The method described in this manuscript is a robust approach to hemp sample preparation which allows for accurate and reproducible downstream molecular analysis of each sample's cannabinoid profile.

\section{Conclusions}

Herein, we are proposing a methodology for hemp sample preparation and homogenization that provides uniform homogenization of a sample, while maintaining the integrity and proportionality of the cannabinoids for downstream molecular analysis. Through use of the active grinding media and bead-mill homogenization, we prepared samples with a majority of the particles $<1.25 \mathrm{~mm}$, resulting in increased cannabinoid recovery when compared to larger particle size homogenates, and no alterations in the carboxylation profiles of CBDA or THCA during processing. This method provides critical reproducibility to processing hemp that will allow the end user to maintain the highest of potency and purity standards moving forward with their molecular analysis.

Acknowledgements The authors would like to acknowledge Karl Jahn, Pete Tortorelli, and Erik Masefield of Omni International, Inc for their financial commitment and continued support of our research. Additionally, we would like to acknowledge Rachele Capobianco and Derick Gordon for their support in establishing the collaborations needed for this project. Finally, we would like to acknowledge Rachel True, Rachel Nash, and Leah Proctor for their sacrifices and unwavering support of our research. IANAFS.

Authors contribution ZPM: formal analysis, writing-original draft, review and editing, visualization. GLR: investigation, formal analysis, writing - review and editing. CMP: methodology, investigation, writing-review and editing. AO: investigation. WT: methodology, investigation. DB: methodology, writing-review and editing, supervision, project administration. TW: investigation. SP: resources, supervision, funding acquisition. BM: investigation. VC investigation. BE: conceptualization, methodology, investigation, data curation, writing-reviewing and editing. JA: conceptualization, supervision, funding acquisition. RJN: conceptualization, investigation, resources, writing - original draft, review and editing, visualization, supervision, project administration, funding acquisition. YCP: conceptualization, investigation, resources, data curation, visualization, supervision, project administration.
Funding This project was funded in its entirety by the private entities Omni International Inc and AMERICANNA Laboratories LLC.

Availability of data and materials All data used in the development of this manuscript is available through email requests to authors ZP Morehouse or RJ Nash at zmorehouse@omni-inc.com or rnash@ omni-inc.com respectively.

\section{Declarations}

Conflict of interest GL Ryan, CM Proctor, A Okparanta,, W Todd, D Bunting, TWhite, B Easparro and J Atwood are all employed by Omni International Inc but do not have any personal financial interests in the success of the company. ZP Morehouse has a consultancy contract with Omni International Inc but does not have any personal financial interests in the success of the company. RJ Nash has a consultancy contract with Omni International but does not have any personal financial interests in the success of the company, and is cofounder and owner of Jeevan Biosciences (Tucker, GA USA) to which he does have financial interests in its success. B Miller, V Colon, and YC Park are all employed by AMERICANNA Laboratories LLC and do not have any personal financial interests in its success. $S$ Perez is cofounder and co-owner of AMERICANNA Laboratories LLC to which he does have personal financial interests in its success.

Ethical approval This manuscript does not report on or involve the use of any animal or human data or tissues, thus ethics approval and consent to participate is not applicable.

Consent for publication This manuscript does not contain data from an individual person, thus consent for publication is not applicable.

Open Access This article is licensed under a Creative Commons Attribution 4.0 International License, which permits use, sharing, adaptation, distribution and reproduction in any medium or format, as long as you give appropriate credit to the original author(s) and the source, provide a link to the Creative Commons licence, and indicate if changes were made. The images or other third party material in this article are included in the article's Creative Commons licence, unless indicated otherwise in a credit line to the material. If material is not included in the article's Creative Commons licence and your intended use is not permitted by statutory regulation or exceeds the permitted use, you will need to obtain permission directly from the copyright holder. To view a copy of this licence, visit http://creativecommons. org/licenses/by/4.0/.

\section{References}

1. Russo EB (2007) History of cannabis and its preparations in saga, science, and sobriquet. Chem Biodivers 4(8):1614-1648. https://doi.org/10.1002/cbdv.200790144

2. Devsi A, Kiyota B, Ouellette T, Hegle AP, Rivera-Acevedo RE, Wong J, Dong Y, Pugsley MK, Fung T (2020) A pharmacological characterization of Cannabis sativa chemovar extracts. J Cannabis Res 2:17. https://doi.org/10.1186/s42238-020-00026-0

3. Amin MR, Ali DW (2019) Pharmacology of medical Cannabis. Adv Exp Med Biol 1162:151-165. https://doi.org/10.1007/ 978-3-030-21737-2 8

4. Ebbert JO, Scharf EL, Hurt RT (2018) Medical Cannabis. Mayo Clin Proc 93(12):1842-1847. https://doi.org/10.1016/j.mayocp. 2018.09.005 
5. Kumar N, Puljević C, Ferris J et al (2019) Cannabis use patterns at the dawn of US cannabis reform. J Cannabis Res 1:5. https:// doi.org/10.1186/s42238-019-0003-z

6. Abuhasira R, Shbiro L, Landschaft Y (2018) Medical use of cannabis and cannabinoids containing products - regulations in Europe and North America. Eur J Intern Med 49:2-6. https:// doi.org/10.1016/j.ejim.2018.01.001

7. Coogan TA (2019) Analysis of the cannabinoid content of strains available in the New Jersey Medicinal Marijuana Program. J Cannabis Res 1:11. https://doi.org/10.1186/ s42238-019-0011-z

8. Mead A (2017) The legal status of cannabis (marijuana) and cannabidiol (CBD) under US law. Epilepsy Behav 70:288-291. https://doi.org/10.1016/j.yebeh.2016.11.021

9. Atkins PL (2019) Sample processing and preparation considerations for solid Cannabis products. J AOAC Int 102(2):427-433. https://doi.org/10.5740/jaoacint.18-0203
10. Pacifici R, Marchei E, Salvatore F, Guandalini L, Busardò FP, Pichini $S$ (2017) Evaluation of cannabinoids concentration and stability in standardized preparations of cannabis tea and cannabis oil by ultra-high performance liquid chromatography tandem mass spectrometry. Clin Chem Lab Med 55(10):1555-1563. https:// doi.org/10.1515/cclm-2016-1060

11. Ibrahim EA, Wang M, Radwan MM et al (2019) Analysis of Terpenes in Cannabis sativa L. Using GC/MS: method development, validation, and application. Planta Med 85(5):431-438. https:// doi.org/10.1055/a-0828-8387

Publisher's Note Springer Nature remains neutral with regard to jurisdictional claims in published maps and institutional affiliations. 